JOURNAL OF

SYMPLECTIC GEOMETRY

Volume 8, Number 2, 225-238, 2010

\title{
DISCRETE HAMILTON-PONTRYAGIN MECHANICS AND GENERATING FUNCTIONS ON LIE GROUPOIDS
}

\author{
ARI STERN
}

\begin{abstract}
We present a discrete analog of the recently introduced HamiltonPontryagin variational principle in Lagrangian mechanics. This unifies two, previously disparate approaches to discrete Lagrangian mechanics: either using the discrete Lagrangian to define a finite version of Hamilton's action principle, or treating it as a symplectic generating function. This is demonstrated for a discrete Lagrangian defined on an arbitrary Lie groupoid; the often encountered special case of the pair groupoid (or Cartesian square) is also given as a worked example.
\end{abstract}

\section{Introduction}

In a recent paper, Yoshimura and Marsden [20] introduced the HamiltonPontryagin variational principle for Lagrangian mechanics. Given a smooth configuration manifold $Q$ and a Lagrangian $L: T Q \rightarrow \mathbb{R}$, this principle defines an action for paths in the so-called Pontryagin bundle $T Q \oplus T^{*} Q$, whose elements are written $(q, v, p)$. Critical paths for this action, in addition to satisfying the usual Euler-Lagrange equations $\dot{p}=\partial L(q, v) / \partial q$, also satisfy the the Legendre transform $p=\partial L(q, v) / \partial v$ and the second-order curve condition $\dot{q}=v$. This can be seen as a unification of two equivalent, but previously disparate, approaches to Lagrangian mechanics, where the Lagrangian is used either (a) to define Hamilton's action functional, studying its critical paths, or (b) to define the Legendre transform, using it to pull back the canonical symplectic structure from the cotangent bundle to the tangent bundle.

There is a similar dilemma in the approach to discrete Lagrangian mechanics. Given a discrete Lagrangian $L_{h}: Q \times Q \rightarrow \mathbb{R}$ (or more generally, $L_{h}: G \rightarrow \mathbb{R}$, where $G \rightrightarrows Q$ is a Lie groupoid), one can either (a) use $L_{h}$ to define a discrete version of Hamilton's action principle, or (b) treat $L_{h}$ as a symplectic generating function. While some progress has been made 
towards combining these approaches into a discrete Hamilton-Pontryagin principle for certain important special cases - namely, the pair groupoid $Q \times Q$ when $Q$ is either a vector space [5,6] or a Lie group [1] — this problem has not yet been resolved for general configuration manifolds $Q$, nor for the even more general case of a Lie groupoid $G \rightrightarrows Q$.

In this paper, we present a discrete Hamilton-Pontryagin principle, which is shown to unify these two disparate approaches (variational principles vs. generating functions) for discrete Lagrangian mechanics on arbitrary Lie groupoids. We begin, in Section 2, by giving a brief review of Lagrangian mechanics, including the continuous Hamilton-Pontryagin principle, as well as summarizing the existing frameworks for discrete Lagrangian mechanics on $Q \times Q$ and on Lie groupoids $G \rightrightarrows Q$. Next, in Section 3, we introduce the discrete Hamilton-Pontryagin principle, which is defined with respect to paths in the cotangent groupoid $T^{*} G \rightrightarrows A^{*} G$ beginning at the zero section; the approach is related to that used by Milinković [11] in studying the Morse homology of generating functions. This variational principle and its solutions, which imply those of the previous approaches to discrete Lagrangian mechanics, are derived first in cotangent bundle coordinates and then given intrinsically. Finally, in Section 4, we work out the special case of the pair groupoid $Q \times Q$, in particular showing how this corresponds to the formulation of Leok and Ohsawa [6] when $Q$ is a vector space.

\section{Lagrangian mechanics, continuous and discrete}

2.1. Lagrangian and Hamilton-Pontryagin mechanics. Let $Q$ be a smooth configuration manifold, and $L: T Q \rightarrow \mathbb{R}$ be a Lagrangian on its tangent bundle. There are two theoretically equivalent, but conceptually distinct, approaches to the Lagrangian mechanics of this system. The first, which we will call the variational approach, is to study critical paths $q$ : $[a, b] \rightarrow Q$ for the action functional

$$
S(q)=\int_{a}^{b} L(q(t), \dot{q}(t)) d t .
$$

Such a path is critical if and only if it satisfies the Euler-Lagrange equations

$$
\frac{d}{d t} \frac{\partial L}{\partial \dot{q}}=\frac{\partial L}{\partial q} \text {. }
$$

The second approach, which we will call the symplectic approach, is to use the Legendre transform $\mathbb{F} L: T Q \rightarrow T^{*} Q$, which takes $(q, v) \mapsto(q, \partial L / \partial v)$, to pull back the canonical symplectic structure from $T^{*} Q$ to $T Q$. If $\omega$ is the canonical symplectic 2-form on $T^{*} Q$, then one can define a 2 -form $\omega_{L}=(\mathbb{F} L)^{*} \omega$ on $T Q$. (The form $\omega_{L}$ is symplectic when $L$ is a hyperreg- 
ular Lagrangian, and presymplectic more generally.) Next, one defines the energy function $E: T Q \rightarrow \mathbb{R}$ given by

$$
E(q, v)=\mathbb{F} L(q, v) v-L(q, v) .
$$

Finally, then, one looks for vector fields $X \in \mathfrak{X}(T Q)$ that satisfy

$$
i_{X} \omega_{L}=d E
$$

which is essentially the tangent bundle version of Hamilton's equations on $T^{*} Q$. (For further background, see [9, Chapter 7].)

Yoshimura and Marsden [20] showed that these two approaches can be unified through an expanded variational principle, which they call the Hamilton-Pontryagin principle. Given a path $(q, v, p):[a, b] \rightarrow T Q \oplus T^{*} Q$, the Hamilton-Pontryagin action is given by

$$
\tilde{S}(q, v, p)=\int_{a}^{b}[L(q(t), v(t))+p(t)(\dot{q}(t)-v(t))] d t .
$$

This is essentially the usual action functional - except, rather than simply prescribing the second-order curve constraint $\dot{q}=v$, one treats $q$ and $v$ as independent variables and then uses $p$ as a Lagrange multiplier to enforce this constraint. Varying over paths with prescribed endpoints, so that $\delta q(a)=0$ and $\delta q(b)=0$, the variation of the action is

$$
\begin{aligned}
d \tilde{S} & (q, v, p)(\delta q, \delta v, \delta p) \\
& =\int_{a}^{b}\left[\frac{\partial L}{\partial q} \delta q+\frac{\partial L}{\partial v} \delta v+p(\delta \dot{q}-\delta v)+\delta p(\dot{q}-v)\right] d t \\
& =\int_{a}^{b}\left[\left(\frac{\partial L}{\partial q}-\dot{p}\right) \delta q+\left(\frac{\partial L}{\partial v}-p\right) \delta v+\delta p(\dot{q}-v)\right] d t
\end{aligned}
$$

Therefore, $(q, v, p)$ is a critical path if and only if it solves the so-called implicit Euler-Lagrange equations

$$
\dot{p}=\frac{\partial L}{\partial q}, \quad p=\frac{\partial L}{\partial v}, \quad \dot{q}=v,
$$

which combine the Euler-Lagrange equations, the Legendre transform, and the second-order curve condition into a single set of equations. It should be noted that this principle is especially useful for studying constrained and other degenerate systems, and is closely connected with the generalized Legendre transform of Tulczyjew [15, 16], the generalized Hamiltonian dynamics formalism of Skinner and Rusk [13], and the Dirac structures of Courant [3]. 
2.2. Discrete Lagrangian mechanics. The idea of discrete Lagrangian mechanics was put forward in seminal papers by Suris [14] and Moser and Veselov [12], among others, and a general theory was developed over the subsequent decade. (See [10], for a comprehensive survey.) This work was motivated by the need to develop structure-preserving (e.g., symplectic) numerical integrators for Lagrangian mechanical systems on general configuration manifolds; the methods developed using this discrete Lagrangian framework are called variational integrators.

For most of the work in this field, the starting point is to replace the Lagrangian $L: T Q \rightarrow \mathbb{R}$ by a discrete Lagrangian $L_{h}: Q \times Q \rightarrow \mathbb{R}$, which approximates the contribution to the action integral,

$$
L_{h}\left(q_{0}, q_{1}\right) \approx \int_{t_{0}}^{t_{1}} L(q(t), \dot{q}(t)) d t
$$

for a time step of size $h=t_{1}-t_{0}$. As with continuous Lagrangian mechanics, there are two typical ways to proceed, following either the variational or the symplectic point of view.

The variational approach to discrete Lagrangian mechanics is as follows. Suppose that we specify a sequence of time steps $a=t_{0}<t_{1}<\cdots<t_{N}=b$, with equal step size $h=t_{n+1}-t_{n}$ for $n=0, \ldots, N-1$. We then define a discrete path to be a sequence of configuration points $q_{0}, \ldots, q_{N} \in Q$; this can be thought of as approximating a continuous path $q:[a, b] \rightarrow Q$, with $q_{n} \approx q\left(t_{n}\right)$. Given the discrete Lagrangian $L_{h}: Q \times Q \rightarrow \mathbb{R}$, the discrete action sum is defined to be

$$
S_{h}\left(q_{0}, \ldots, q_{N}\right)=\sum_{n=0}^{N-1} L_{h}\left(q_{n}, q_{n+1}\right) \approx \int_{a}^{b} L(q(t), \dot{q}(t)) d t .
$$

Next, taking fixed-endpoint variations of the discrete path, so that $\delta q_{0}=0$ and $\delta q_{N}=0$, it follows that

$$
d S_{h}\left(q_{0}, \ldots, q_{N}\right)\left(\delta q_{0}, \ldots, \delta q_{N}\right)=\sum_{n=1}^{N-1}\left[\partial_{0} L_{h}\left(q_{n}, q_{n+1}\right)+\partial_{1} L_{h}\left(q_{n-1}, q_{n}\right)\right] \delta q_{n} .
$$

Therefore, a discrete path is critical if and only if it satisfies the discrete Euler-Lagrange equations

$$
\partial_{0} L_{h}\left(q_{n}, q_{n+1}\right)+\partial_{1} L_{h}\left(q_{n-1}, q_{n}\right)=0, \quad n=1, \ldots, N-1 .
$$

This implicitly defines a two-step numerical integrator on $Q \times Q$, which (given suitable assumptions of nondegeneracy) maps $\left(q_{n-1}, q_{n}\right) \mapsto\left(q_{n}, q_{n+1}\right)$.

On the other hand, the symplectic approach to discrete Lagrangian mechanics is to view $L_{h}: Q \times Q \rightarrow \mathbb{R}$ as the generating function for a 
symplectic map on $T^{*} Q$. To do this, one defines the discrete Legendre transforms $\mathbb{F}^{ \pm} L_{h}: Q \times Q \rightarrow T^{*} Q$ by

$$
\mathbb{F}^{-} L_{h}\left(q_{0}, q_{1}\right)=-\partial_{0} L_{h}\left(q_{0}, q_{1}\right), \quad \mathbb{F}^{+} L_{h}\left(q_{0}, q_{1}\right)=\partial_{1} L_{h}\left(q_{0}, q_{1}\right) .
$$

Therefore, we can implicitly define a map on $T^{*} Q$ by

$$
p_{0}=\mathbb{F}^{-} L_{h}\left(q_{0}, q_{1}\right), \quad p_{1}=\mathbb{F}^{+} L_{h}\left(q_{0}, q_{1}\right),
$$

and if $\mathbb{F}^{-} L_{h}$ is invertible, this defines one step of the symplectic integrator

$$
\mathbb{F}^{+} L_{h} \circ\left(\mathbb{F}^{-} L_{h}\right)^{-1}: T^{*} Q \rightarrow T^{*} Q, \quad\left(q_{0}, p_{0}\right) \mapsto\left(q_{1}, p_{1}\right) .
$$

More precisely, the discrete Legendre transforms define the Lagrangian submanifold of $\left(T^{*} Q,-\omega\right) \times\left(T^{*} Q, \omega\right)$ generated by $L_{h}$. From this perspective, if the invertibility condition holds, then this submanifold is the graph of a symplectic map on $T^{*} Q$ [see 17,18$]$.

Note that, if we perform the composition in the opposite order, we get the previously derived two-step method,

$$
\left(\mathbb{F}^{-} L_{h}\right)^{-1} \circ \mathbb{F}^{+} L_{h}: Q \times Q \rightarrow Q \times Q, \quad\left(q_{n-1}, q_{n}\right) \mapsto\left(q_{n}, q_{n+1}\right),
$$

where the discrete Euler-Lagrange equations follow automatically from the fact that $\mathbb{F}^{-} L_{h}\left(q_{n}, q_{n+1}\right)=\mathbb{F}^{+} L_{h}\left(q_{n-1}, q_{n}\right)$.

2.3. Discrete Lagrangian mechanics and Lie groupoids. Weinstein [19] observed that both approaches in the previous section can be generalized using Lie groupoids. Let $G \rightrightarrows Q$ be a given Lie groupoid, and define a discrete Lagrangian $L_{h}: G \rightarrow \mathbb{R}$. The earlier formulations then coincide with the special case $G=Q \times Q$, which is called the pair groupoid. This perspective has continued to bear fruit in recent years, being further developed by Marrero et al. [8] and extended to discrete nonholonomic Lagrangian mechanics by Iglesias et al. [4].

In the variational approach, one begins by taking a fixed element $g \in G$ and considering the space of admissible sequences, which consist of composable elements $g_{1}, \ldots, g_{N} \in G$ such that $g_{1} \cdots g_{N}=g$. The discrete action for an admissible sequence is then taken to be

$$
S_{h}\left(g_{1}, \ldots, g_{N}\right)=\sum_{n=1}^{N} L_{h}\left(g_{n}\right),
$$

and discrete Euler-Lagrange equations are obtained by finding sequences that are critical for this action function. In the case of the pair groupoid $Q \times Q$, fixing an element of the groupoid corresponds simply to fixing the endpoints $g=\left(q_{0}, q_{N}\right)$, while the set of admissible sequences $\left(q_{0}, q_{1}\right), \ldots,\left(q_{N-1}, q_{N}\right) \in Q \times Q$ can be identified with the sequence of configuration points $q_{0}, \ldots, q_{N} \in Q$. 
Marrero et al. [8] showed that the discrete Euler-Lagrange equations can be expessed in terms of left- and right-invariant vector fields on $G$, each of which can be identified with sections of the Lie algebroid $A G \rightarrow Q$ associated to $G$. To describe this, we must first introduce some notation. Let $\alpha, \beta: G \rightarrow Q$ denote the source and target maps, $\epsilon: Q \rightarrow G$ denote the identity section, and $i: G \rightarrow G$ denote the inversion map on $G$. For any $g \in G$, define the left- and right-translation maps, respectively, by

$$
\begin{aligned}
\ell_{g}: \alpha^{-1}(\beta(g)) & \rightarrow \alpha^{-1}(\alpha(g)) & r_{g}: \beta^{-1}(\alpha(g)) & \rightarrow \beta^{-1}(\beta(g)) \\
g^{\prime} & \mapsto g g^{\prime} & g^{\prime} & \mapsto g^{\prime} g .
\end{aligned}
$$

Then, given a section $X \in \Gamma(A G)$, the left-invariant vector field $\overleftarrow{X} \in \mathfrak{X}(G)$ is defined by $\overleftarrow{X}(g)=T_{\epsilon(\beta(g))} \ell_{g}(X(\beta(g)))$. Similarly, the right-invariant vector field $\vec{X} \in \mathfrak{X}(G)$ is given by $\vec{X}(g)=-\left(T_{\epsilon(\alpha(g))}\left(r_{g} \circ i\right)\right)(X(\alpha(g)))$. This implies the following relationship between the brackets $\llbracket \cdot, \cdot \rrbracket$ on $\Gamma(A G)$ and $[\cdot, \cdot]$ on $\mathfrak{X}(G)$ :

$$
\overleftarrow{\llbracket X, Y \rrbracket}=[\overleftarrow{X}, \overleftarrow{Y}], \quad \overrightarrow{\llbracket X, Y \rrbracket}=-[\vec{X}, \vec{Y}]
$$

With these definitions, the discrete Euler-Lagrange equations are

$$
\overleftarrow{X}\left[L_{h}\right]\left(g_{n}\right)=\vec{X}\left[L_{h}\right]\left(g_{n+1}\right), \quad n=1, \ldots, N-1
$$

for all sections $X \in \Gamma(A G)$. In the pair groupoid case, one sees that

$$
\begin{aligned}
& \overleftarrow{X}\left[L_{h}\right]\left(q_{n-1}, q_{n}\right)=\partial_{1} L_{h}\left(q_{n-1}, q_{n}\right) X\left(q_{n}\right) \\
& \vec{X}\left[L_{h}\right]\left(q_{n}, q_{n+1}\right)=-\partial_{0} L_{h}\left(q_{n}, q_{n+1}\right) X\left(q_{n}\right)
\end{aligned}
$$

for any $X \in \mathfrak{X}(Q)$, so this formulation agrees with the earlier expression of the discrete Euler-Lagrange equations on $Q \times Q$.

For the symplectic approach, we begin by defining the cotangent groupoid $T^{*} G \rightrightarrows A^{*} G$, where the base $A^{*} G \rightarrow Q$ is the dual of the Lie algebroid $A G \rightarrow Q$. This is a symplectic groupoid, with a canonical symplectic structure on $T^{*} G$ and Poisson structure on $A^{*} G$; moreover, the source and target maps are anti-Poisson and Poisson, respectively $[2,7]$. Explicitly, the source map $\tilde{\alpha}: T^{*} G \rightarrow A^{*} G$ and target map $\tilde{\beta}: T^{*} G \rightarrow A^{*} G$ may be defined such that, taking $\mu \in T_{g}^{*} G$,

$$
\tilde{\alpha}(\mu) X(\alpha(g))=\mu \vec{X}(g), \quad \tilde{\beta}(\mu) X(\beta(g))=\mu \overleftarrow{X}(g)
$$

for every section $X \in \Gamma(A G)$. (The multiplicative structure of $T^{*} G$ will not be necessary here, so we omit discussion of it.) 
Now, the discrete Lagrangian $L_{h}: G \rightarrow \mathbb{R}$ generates a Lagrangian submanifold of the cotangent groupoid, $d L_{h}(G) \subset T^{*} G$. If $L_{h}$ is suitably nondegenerate, it follows that this submanifold determines a Poisson automorphism $A^{*} G \rightarrow A^{*} G[19,8]$. Specifically, define the discrete Legendre transforms $\mathbb{F}^{ \pm} L_{h}: G \rightarrow A^{*} G$ by

$$
\mathbb{F}^{-} L_{h}=\tilde{\alpha} \circ d L_{h}, \quad \mathbb{F}^{+} L_{h}=\tilde{\beta} \circ d L_{h} .
$$

Therefore, $\left\{\left(\mathbb{F}^{-} L_{h}(g), \mathbb{F}^{+} L_{h}(g)\right) \mid g \in G\right\}$ is a coisotropic relation on $A^{*} G$, and if $\mathbb{F}^{-} L_{h}$ is a diffeomorphism, then this relation is the graph of the discrete flow map

$$
\mathbb{F}^{+} L_{h} \circ\left(\mathbb{F}^{-} L_{h}\right)^{-1}: A^{*} G \rightarrow A^{*} G .
$$

To see that this is compatible with the discrete Euler-Lagrange equations, observe that for any $X \in \Gamma(A G)$,

$$
\begin{gathered}
\left(\tilde{\alpha} \circ d L_{h}\right)(g) X(\alpha(g))=d L_{h}(g) \vec{X}(g)=\vec{X}\left[L_{h}\right](g) \\
\left(\tilde{\beta} \circ d L_{h}\right)(g) X(\beta(g))=d L_{h}(g) \overleftarrow{X}(g)=\overleftarrow{X}\left[L_{h}\right](g)
\end{gathered}
$$

Thus, the discrete Euler-Lagrange equations correspond to the condition $\mathbb{F}^{+} L_{h}\left(g_{n}\right)=\mathbb{F}^{-} L_{h}\left(g_{n+1}\right)$. Equivalently, this means that $g_{1}, \ldots, g_{N}$ is a solution of the discrete Euler-Lagrange equations when $d L_{h}\left(g_{1}\right), \ldots, d L_{h}\left(g_{N}\right)$ is a composable sequence in $T^{*} G$.

\section{The discrete Hamilton-Pontryagin principle}

For the continuous Hamilton-Pontryagin principle, the key idea was to relax the condition for curves in $T Q$ to be second-order, but to enforce this condition weakly using Lagrange multipliers in $T^{*} Q$. Analogously, we will develop a discrete Hamilton-Pontryagin principle by relaxing the requirement that the sequence $g_{1}, \ldots, g_{N} \in G$ is admissible - requiring only that it be admissible up to homotopy - and will weakly enforce the condition that this homotopy is, in fact, constant. To allow for the "Lagrange multipliers," these homotopies will be given by paths in $T^{*} G$ rather than in $G$.

To simplify the exposition, we will first sketch this formulation, in Section 3.1, using cotangent bundle coordinates $(g, \mu) \in T^{*} G$. The fully intrinsic treatment on $T^{*} G$ will be given subsequently, in Section 3.2, along with definitions and the proof of the main theorem.

3.1. Formulation in cotangent bundle coordinates. Suppose that $\left(g_{n}, \mu_{n}\right):[0,1] \rightarrow T^{*} G$, for $n=1, \ldots, N$, is a sequence of paths in the cotangent groupoid. Furthermore, it is required that the initial point $\left(g_{n}(0), \mu_{n}(0)\right)$ of each path lie in the zero section, so that $\mu_{n}(0)=0_{g_{n}(0)}$, and that $g_{1}(0), \ldots, g_{N}(0)$ be an admissible sequence for a fixed element $g \in G$. 
Given such a sequence of paths, define the discrete Hamilton-Pontryagin action to be

$$
\tilde{S}_{h}\left(g_{1}, \mu_{1}, \ldots, g_{N}, \mu_{N}\right)=\sum_{n=1}^{N} \int_{0}^{1}\left[L_{h}\left(g_{n}(s)\right)+\mu_{n}(s) g_{n}^{\prime}(s)\right] d s .
$$

Variations of this action are

$$
\begin{gathered}
d \tilde{S}_{h}\left(g_{1}, \mu_{1}, \ldots, g_{N}, \mu_{N}\right)\left(\delta g_{1}, \delta \mu_{1}, \ldots, \delta g_{N}, \delta \mu_{N}\right) \\
=\sum_{n=1}^{N} \int_{0}^{1}\left[d L_{h}\left(g_{n}(s)\right) \delta g_{n}(s)+\mu_{n}(s) \delta g_{n}^{\prime}(s)+\delta \mu_{n}(s) g_{n}^{\prime}(s)\right] d s \\
=\sum_{n=1}^{N}\left(\int_{0}^{1}\left[\left(d L_{h}\left(g_{n}(s)\right)-\mu_{n}^{\prime}(s)\right) \delta g_{n}(s)+\delta \mu_{n}(s) g_{n}^{\prime}(s)\right] d s\right. \\
\left.\quad+\mu_{n}(1) \delta g_{n}(1)\right) .
\end{gathered}
$$

Now, the integral terms vanish when $\left(g_{n}, \mu_{n}\right)$ is a solution of

$$
\mu_{n}^{\prime}(s)=d L_{h}\left(g_{n}(s)\right), \quad g_{n}^{\prime}(s)=0 .
$$

The second equation states that $g_{n}(s)=g_{n}$ is constant, so $\mu_{n}(s)$ moves vertically along the fiber $T_{g_{n}}^{*} G$, beginning at $\mu_{n}(0)=0_{g_{n}}$. We use this fact to solve the remaining equation,

$$
\mu_{n}(s)=\mu_{n}(0)+s d L_{h}\left(g_{n}\right)=s d L_{h}\left(g_{n}\right),
$$

so in particular, $\mu_{n}(1)=d L_{h}\left(g_{n}\right)$. Additionally, $g_{1}, \ldots, g_{N}$ is an admissible sequence, since $g_{n}=g_{n}(0)$, which was assumed to be admissible. Finally, restricting to $\left(g_{n}, \mu_{n}\right)$ where these equations are satisfied, the variation of the action is given by the remaining boundary terms

$$
\sum_{n=1}^{N} \mu_{n}(1) \delta g_{n}(1)=\sum_{n=1}^{N} d L_{h}\left(g_{n}\right) \delta g_{n}
$$

However, the restricted variations $\delta g_{n}$ are no longer completely arbitrary, since they must be tangent to the space of admissible sequences $g_{1}, \ldots, g_{N}$. Therefore, we are back in the case considered by Marrero et al. [8], so these terms vanish precisely when $g_{n}$ is a solution of the discrete Euler-Lagrange equations - or equivalently, when $\mu_{1}(1), \ldots, \mu_{N}(1) \in T^{*} G$ is a composable sequence.

In summary, $d \tilde{S}_{h}=0$ implies that $g_{1}(1), \ldots, g_{N}(1)$ is an admissible sequence in $G$, that $\mu_{n}(1)=d L_{h}\left(g_{n}(1)\right)$, and that $\mu_{1}(1), \ldots, \mu_{N}(1)$ is a composable sequence in $T^{*} G$. 
3.2. Intrinsic formulation. We will now make precise the approach sketched out in the previous section, doing this intrinsically on $T^{*} G$ rather than in cotangent bundle coordinates. Let $\pi_{G}: T^{*} G \rightarrow G$ be the cotangent bundle projection, $\tilde{\theta}$ be the canonical 1 -form on $T^{*} G$, and $\tilde{\omega}=-d \tilde{\theta}$ be the canonical symplectic 2-form.

Given a Lie groupoid $G \rightrightarrows Q$, define the fibration $E \rightarrow G$ consisting of paths in $T^{*} G$ beginning at the zero section,

$$
E=\left\{\gamma:[0,1] \rightarrow T^{*} G \mid \gamma(0) \in 0_{G}\right\},
$$

where the projection is given by $\gamma \mapsto\left(\pi_{G} \circ \gamma\right)(1)$.

Definition 3.1. Given a fixed $g \in G$, a sequence of paths $\gamma_{1}, \ldots, \gamma_{N} \in E$ is said to be admissible if the initial elements $\left(\pi_{G} \circ \gamma_{1}\right)(0), \ldots,\left(\pi_{G} \circ \gamma_{N}\right)(0)$ form an admissible sequence in $G$.

This implies that the projected sequence $\left(\pi_{G} \circ \gamma_{1}\right)(1), \ldots,\left(\pi_{G} \circ \gamma_{N}\right)(1) \in$ $G$ is only admissible up to homotopy, which is weaker than the usual assumption that $g_{1}, \ldots, g_{N}$ must actually be an admissible sequence.

Definition 3.2. Let $L_{h}: G \rightarrow \mathbb{R}$ be a discrete Lagrangian, and $\gamma_{1}, \ldots, \gamma_{N} \in$ $E$ be an admissible sequence of paths. Then the discrete HamiltonPontryagin action of this sequence is

$$
\tilde{S}_{h}\left(\gamma_{1}, \ldots, \gamma_{N}\right)=\sum_{n=1}^{N}\left[\int_{0}^{1}\left(L_{h} \circ \pi_{G}\right)\left(\gamma_{n}(s)\right) d s+\int_{\gamma_{n}} \tilde{\theta}\right]
$$

The sequence is said to satisfy the discrete Hamilton-Pontragin principle if

$$
d \tilde{S}_{h}\left(\gamma_{1}, \ldots, \gamma_{N}\right)=0 .
$$

Theorem 3.1. Let $\gamma_{1}, \ldots, \gamma_{N} \in E$ be an admissible sequence of paths, and denote $\mu_{n}=\gamma_{n}(1) \in T^{*} G$ and $g_{n}=\pi_{G}\left(\mu_{n}\right) \in G$ for $n=1, \ldots, N$. If the sequence satisfies the Hamilton-Pontryagin principle, then

(i) $g_{1}, \ldots, g_{N}$ is an admissible sequence in $G$,

(ii) $\mu_{n}=d L_{h}\left(g_{n}\right)$ for $n=1, \ldots, N$,

(iii) $\mu_{1}, \ldots, \mu_{N}$ is a composable sequence in $T^{*} G$.

These properties may be thought of as, respectively, (i) the discrete second-order curve condition; (ii) the discrete Legendre transform; and (iii) the discrete Euler-Lagrange equations.

Proof. Take a variation of the Hamilton-Pontryagin action to obtain

$$
d \tilde{S}_{h}\left(\gamma_{1}, \ldots, \gamma_{N}\right)\left(\delta \gamma_{1}, \ldots, \delta \gamma_{N}\right)=\sum_{n=1}^{N}\left[\int_{0}^{1}\left(\pi_{G}^{*} d L_{h}\right) \delta \gamma_{n}(s) d s+\int_{\gamma_{n}} \mathfrak{L}_{\delta \gamma_{n}} \tilde{\theta}\right]
$$


Applying Cartan's "magic formula," the Lie derivative terms become

$$
\begin{aligned}
\int_{\gamma_{n}} \mathfrak{L}_{\delta \gamma_{n}} \tilde{\theta} & =\int_{\gamma_{n}}\left(i_{\delta \gamma_{n}} d \tilde{\theta}+d i_{\delta \gamma_{n}} \tilde{\theta}\right) \\
& =-\int_{\gamma_{n}} i_{\delta \gamma_{n}} \tilde{\omega}+\int_{\partial \gamma_{n}} \tilde{\theta} \delta \gamma_{n} \\
& =\int_{0}^{1} i_{\gamma_{n}^{\prime}(s)} \tilde{\omega} \delta \gamma_{n}(s) d s+\tilde{\theta} \delta \gamma_{n}(1) .
\end{aligned}
$$

Therefore, the variation of the action is

$$
\begin{aligned}
& d \tilde{S}_{h}\left(\gamma_{1}, \ldots, \gamma_{N}\right)\left(\delta \gamma_{1}, \ldots, \delta \gamma_{N}\right) \\
& \quad=\sum_{n=1}^{N}\left[\int_{0}^{1}\left(\pi_{G}^{*} d L_{h}+i_{\gamma_{n}^{\prime}(s)} \tilde{\omega}\right) \delta \gamma_{n}(s) d s+\tilde{\theta} \delta \gamma_{n}(1)\right]
\end{aligned}
$$

For variations taken along the fibers of $E$, this is stationary when

$$
i_{\gamma_{n}^{\prime}(s)} \tilde{\omega}=-\pi_{G}^{*} d L_{h}, \quad n=1, \ldots, N
$$

i.e., the paths $\gamma_{n}$ are solutions to Hamilton's equations for the singular Hamiltonian $\tilde{H}=-L_{h} \circ \pi_{G}: T^{*} G \rightarrow \mathbb{R}$. However, since $\tilde{H}$ is constant on fibers of $T^{*} G$ (which form a Lagrangian foliation), the Hamiltonian flow is fiber-preserving, and hence the projection $\left(\pi_{G} \circ \gamma_{n}\right)(s)=g_{n}$ is constant. This proves $(\mathrm{i})$, since $g_{n}=\left(\pi_{G} \circ \gamma_{n}\right)(0)$, which was assumed to be an admissible sequence in $G$. Furthermore, the Hamiltonian vector field is constant on fibers of $T^{*} G$, and it follows that $\gamma_{n}(s)=s d L_{h}\left(g_{n}\right)$. Therefore, $\mu_{n}=\gamma_{n}(1)=d L_{h}\left(g_{n}\right)$, which proves (ii).

Finally, restricting the action to these solutions, the integral terms vanish, and the remaining boundary terms are

$$
\sum_{n=1}^{N} \tilde{\theta} \delta \gamma_{n}(1)=\sum_{n=1}^{N} \mu_{n} \delta g_{n}=\sum_{n=1}^{N} d L_{h}\left(g_{n}\right) \delta g_{n} .
$$

This is precisely the variation of the usual discrete action, so these terms vanish when the discrete Euler-Lagrange equations are satisfied. Therefore, $\tilde{\beta}\left(\mu_{n}\right)=\tilde{\alpha}\left(\mu_{n+1}\right)$ for $n=1, \ldots, N-1$, so $\mu_{1}, \ldots, \mu_{N}$ is a composable sequence in $T^{*} G$, which completes the proof of (iii).

Remark 3.1. For a single time step $N=1$, the action

$$
\tilde{S}_{h}: E \rightarrow \mathbb{R}, \quad \gamma \mapsto \int_{0}^{1}\left(L_{h} \circ \pi_{G}\right)(\gamma(s)) d s+\int_{\gamma} \tilde{\theta}
$$

can be understood as a Morse family, which generates the usual Lagrangian submanifold $d L_{h}(G) \subset T^{*} G$. This is closely related to the work of Milinković [11], who studied a similar action principle on $T^{*} Q$, in connection with the Morse homology of generating functions for Lagrangian submanifolds. In 
Milinković's formulation, the Lagrangian submanifold is determined by the time-1 Hamiltonian isotopy of $\tilde{H}$, which, in this case, takes the zero section to $d L_{h}(G)$.

\section{Example: the pair groupoid}

Let $G=Q \times Q$ be the pair groupoid, and $L_{h}: Q \times Q \rightarrow \mathbb{R}$ be a discrete Lagrangian. Fixing the endpoints $g=\left(q_{0}, q_{N}\right)$, suppose that $\gamma_{1}, \ldots, \gamma_{N} \in E$ is an admissible sequence of paths, where $\gamma_{n}(s)=\left(q_{n}^{0}(s), p_{n}^{0}(s), q_{n}^{1}(s), p_{n}^{1}(s)\right)$. Since the sequence is admissible, this implies in particular that $q_{0}=q_{1}^{0}(0)$, $q_{n}^{1}(0)=q_{n+1}^{0}(0)$ for $n=1, \ldots, N-1$, and $q_{N}^{1}(0)=q_{N}$. The discrete Hamilton-Pontryagin action of this sequence is then

$$
\tilde{S}_{h}\left(\gamma_{1}, \ldots, \gamma_{N}\right)=\sum_{n=1}^{N} \int_{0}^{1}\left[L_{h}\left(q_{n}^{0}(s), q_{n}^{1}(s)\right)-p_{n}^{0}(s) q_{n}^{0 \prime}(s)+p_{n}^{1}(s) q_{n}^{1^{\prime}}(s)\right] d s .
$$

Taking variations of this action gives

$$
\begin{aligned}
& d \tilde{S}_{h}\left(\gamma_{1}, \ldots, \gamma_{N}\right)\left(\delta \gamma_{1}, \ldots, \delta \gamma_{N}\right) \\
& =\sum_{n=1}^{N} \int_{0}^{1}\left[\partial_{0} L_{h}\left(q_{n}^{0}(s), q_{n}^{1}(s)\right) \delta q_{n}^{0}(s)+\partial_{1} L_{h}\left(q_{n}^{0}(s), q_{n}^{1}(s)\right) \delta q_{n}^{1}(s)\right. \\
& \left.\quad-p_{n}^{0}(s) \delta q_{n}^{0 \prime}(s)+p_{n}^{1}(s) \delta q_{n}^{1^{\prime}}(s)-\delta p_{n}^{0}(s) q_{n}^{0 \prime}(s)+\delta p_{n}^{1}(s) q_{n}^{1^{\prime}}(s)\right] d s,
\end{aligned}
$$

and integrating by parts, this simplifies to

$$
\begin{gathered}
d \tilde{S}_{h}\left(\gamma_{1}, \ldots, \gamma_{N}\right)\left(\delta \gamma_{1}, \ldots, \delta \gamma_{N}\right) \\
=\sum_{n=1}^{N}\left(\int _ { 0 } ^ { 1 } \left[\left(\partial_{0} L_{h}\left(q_{n}^{0}(s), q_{n}^{1}(s)\right)+p_{n}^{0 \prime}(s)\right) \delta q_{n}^{0}(s)\right.\right. \\
+\left(\partial_{1} L_{h}\left(q_{n}^{0}(s), q_{n}^{1}(s)\right)-p_{n}^{1 \prime}(s)\right) \delta q_{n}^{1}(s) \\
\left.-\delta p_{n}^{0}(s) q_{n}^{0 \prime}(s)+\delta p_{n}^{1}(s) q_{n}^{1^{\prime}}(s)\right] d s \\
\left.-p_{n}^{0}(1) \delta q_{n}^{0}(1)+p_{n}^{1}(1) \delta q_{n}^{1}(1)\right)
\end{gathered}
$$

For variations taken along the fibers of $E$, this is stationary when the integral terms vanish, so

$$
\begin{aligned}
& p_{n}^{0 \prime}(s)=-\partial_{0} L_{h}\left(q_{n}^{0}(s), q_{n}^{1}(s)\right), \quad q_{n}^{0 \prime}(s)=0, \\
& p_{n}^{1 \prime}(s)=\partial_{1} L_{h}\left(q_{n}^{0}(s), q_{n}^{1}(s)\right), \quad q_{n}^{1 \prime}(s)=0,
\end{aligned}
$$

for $n=1, \ldots, N$. Therefore, $q_{n}^{0}(s)=q_{n}^{0}$ and $q_{n}^{1}(s)=q_{n}^{1}$ are constant, so

$$
p_{n}^{0}(1)=-\partial_{0} L_{h}\left(q_{n}^{0}, q_{n}^{1}\right), \quad p_{n}^{1}(1)=\partial_{1} L_{h}\left(q_{n}^{0}, q_{n}^{1}\right), \quad n=1, \ldots, N .
$$


Furthermore, the admissibility assumption implies that $q_{0}=q_{1}^{0}, q_{n}^{1}=q_{n+1}^{0}$ for $n=1, \ldots, N-1$, and $q_{N}^{1}=q_{N}$.

Finally, restricting to these solutions, the restricted variations must then satisfy $\delta q_{1}^{0}=0, \delta q_{n}^{1}=\delta q_{n+1}^{0}=$ for $n=1, \ldots, N-1$, and $\delta q_{N}^{1}=0$. Therefore, the remaining terms of the action are

$$
\sum_{n=1}^{N}\left(-p_{n}^{0}(1) \delta q_{n}^{0}+p_{n}^{1}(1) \delta q_{n}^{1}\right)=\sum_{n=1}^{N-1}\left(-p_{n+1}^{0}(1)+p_{n}^{1}(1)\right) \delta q_{n}^{1},
$$

which vanish when $p_{n}^{1}(1)=p_{n+1}^{0}(1)$ for $n=1, \ldots, N-1$.

In summary, if $\gamma_{1}, \ldots, \gamma_{N}$ satisfies the discrete Hamilton-Pontryagin principle on $Q \times Q$, then the following is true:

(i) $q_{0}=q_{1}^{0}, q_{n}^{1}=q_{n+1}^{0}$ for $n=1, \ldots, N-1$, and $q_{N}^{1}=q_{N}$,

(ii) $p_{n}^{0}(1)=-\partial_{0} L_{h}\left(q_{n}^{0}, q_{n}^{1}\right)$ and $p_{n}^{1}(1)=\partial_{1} L_{h}\left(q_{n}^{0}, q_{n}^{1}\right)$ for $n=1, \ldots, N$,

(iii) $p_{n}^{1}(1)=p_{n+1}^{0}(1)$ for $n=1, \ldots, N-1$.

These are, respectively, the discrete second-order curve condition, the discrete Legendre transform, and the discrete Euler-Lagrange equations for systems on $Q \times Q$.

Remark 4.1. The cotangent paths $p_{n}^{0}, p_{n}^{1}$ can be seen as Lagrange multipliers, which serve to enforce the composability conditions $q_{n}^{1}=q_{n+1}^{0}$, as well as the endpoint constraints $q_{1}^{0}=q_{0}$ and $q_{N}^{1}=q_{N}$. Leok and Ohsawa [6] showed that, when $Q$ is a vector space (or given a particular local coordinate neighborhood), this can be done even more directly, by simply subtracting these points rather than taking a path between them. This leads to an alternative choice of the action sum,

$$
\sum_{n=1}^{N} L_{h}\left(q_{n}^{0}, q_{n}^{1}\right)+p_{0}\left(q_{1}^{0}-q_{0}\right)+\sum_{n=1}^{N-1} p_{n}\left(q_{n+1}^{0}-q_{n}^{1}\right)+p_{N}\left(q_{N}^{1}-q_{N}\right),
$$

whose variations are computed to be

$$
\begin{aligned}
& \sum_{n=1}^{N}\left[\left(\partial_{0} L_{h}\left(q_{n}^{0}, q_{n}^{1}\right)+p_{n-1}\right) \delta q_{n}^{0}+\left(\partial_{1} L_{h}\left(q_{n}^{0}, q_{n}^{1}\right)-p_{n}\right) \delta q_{n}^{1}\right] \\
& \quad+\delta p_{0}\left(q_{1}^{0}-q_{0}\right)+\sum_{n=1}^{N-1} \delta p_{n}\left(q_{n+1}^{0}-q_{n}^{1}\right)+\delta p_{N}\left(q_{N}^{1}-q_{N}\right) .
\end{aligned}
$$

Therefore, this vanishes when

$$
p_{n-1}=-\partial_{0} L_{h}\left(q_{n}^{0}, q_{n}^{1}\right), \quad p_{n}=\partial_{1} L_{h}\left(q_{n}^{0}, q_{n}^{1}\right), \quad n=1, \ldots, N
$$

and when

$$
q_{1}^{0}=q_{0}, \quad q_{N}^{1}=q_{N}, \quad q_{n}^{1}=q_{n+1}^{0}, \quad n=1, \ldots, N-1,
$$

which are consistent with the results obtained in this section. 
Effectively, the Leok-Ohsawa approach can be interpreted as using a smaller fibration $E^{\prime}=Q \times Q \times T^{*} Q \times T^{*} Q$, consisting only of the endpoints of the paths in $E$, to define a Morse family over $Q \times Q$. However, when $Q$ is not a vector space, there is no intrinsic, global meaning to subtracting two points in $Q$. This action may still be defined locally, in a neighborhood of the diagonal of $Q \times Q$, given a choice of local coordinates or a retraction. In general, though, one requires the larger space of paths $E$ in order to define the discrete Hamilton-Pontryagin principle globally.

\section{References}

[1] N. Bou-Rabee and J.E. Marsden, Hamilton-Pontryagin integrators on Lie groups part I: Introduction and structure-preserving properties, Found. Comput. Math. 9(2) (2009), 197-219.

[2] A. Coste, P. Dazord and A. Weinstein, Groupoïdes symplectiques, in 'Publications du Département de Mathématiques,' Nouvelle Série. A, Vol. 2, volume 87 of Publ. Dép. Math. Nouvelle Sér. A, i-ii, 1-62, Univ. Claude-Bernard, Lyon, 1987.

[3] T.J. Courant, Dirac manifolds, Trans. Amer. Math. Soc. 319(2) (1990), 631-661.

[4] D. Iglesias, J.C. Marrero, D. Martín de Diego and E. Martínez, Discrete nonholonomic Lagrangian systems on Lie groupoids, J. Nonlinear Sci. 18(3) (2008), 221-276.

[5] L. Kharevych, W. Yang, Y. Tong, E. Kanso, J.E. Marsden, P. Schröder and M. Desbrun, Geometric, variational integrators for computer animation, in SCA '06: Proceedings of the 2006 ACM SIGGRAPH/Eurographics symposium on Computer animation, 43-51, Eurographics Association, Aire-la-Ville, Switzerland, 2006.

[6] M. Leok and T. Ohsawa, Discrete Dirac structures and variational discrete Dirac mechanics, 2008, Preprint, arXiv:0810.0740 [math.SG].

[7] C.-M. Marle, From momentum maps and dual pairs to symplectic and Poisson groupoids, in The breadth of symplectic and Poisson geometry, Vol. 232 of Progr. Math., 493-523, Birkhäuser Boston, Boston, MA, 2005.

[8] J.C. Marrero, D. Martín de Diego and E. Martínez, Discrete Lagrangian and Hamiltonian mechanics on Lie groupoids, Nonlinearity, 19(6) (2006), 1313-1348.

[9] J.E. Marsden and T.S. Ratiu, Introduction to mechanics and symmetry, Vol. 17 of Texts in Applied Mathematics, 2nd ed., Springer-Verlag, New York, 1999.

[10] J.E. Marsden and M. West, Discrete mechanics and variational integrators, Acta Numer. 10 (2001), 357-514.

[11] D. Milinković, Morse homology for generating functions of Lagrangian submanifolds, Trans. Amer. Math. Soc. 351(10) (1999), 3953-3974.

[12] J. Moser and A.P. Veselov, Discrete versions of some classical integrable systems and factorization of matrix polynomials, Comm. Math. Phys. 139(2) (1991), 217-243.

[13] R. Skinner and R. Rusk, Generalized Hamiltonian dynamics. I. Formulation on $T^{*} Q \oplus$ TQ, J. Math. Phys. 24(11) (1983), 2589-2594.

[14] Y.B. Suris, Hamiltonian methods of Runge-Kutta type and their variational interpretation, Mat. Model. 2(4) (1990), 78-87.

[15] W.M. Tulczyjew, Hamiltonian systems, Lagrangian systems and the Legendre transformation, in Symposia Mathematica, Vol. XIV (Convegno di Geometria Simplettica e Fisica Matematica, INDAM, Rome, 1973), 247-258, Academic Press, London, 1974.

[16] W.M. Tulczyjew, The Legendre transformation, Ann. Inst. H. Poincaré Sect. A (N.S.), 27(1) (1977), 101-114. 
[17] A. Weinstein, Symplectic manifolds and their Lagrangian submanifolds, Adv. Math. 6 (1971), 329-346.

[18] A. Weinstein, Lectures on symplectic manifolds, Vol. 29 of CBMS Regional Conference Series in Mathematics, Am. Math. Soc., Providence, RI, 1979.

[19] A. Weinstein, Lagrangian mechanics and groupoids, in Mechanics day (Waterloo, ON, 1992), Vol. 7 of Fields Inst. Commun., 207-231, Am. Math. Soc., Providence, RI, 1996.

[20] H. Yoshimura and J.E. Marsden, Dirac structures in Lagrangian mechanics, II, Variational structures, J. Geom. Phys. 57(1) (2006), 209-250.

Department of Mathematics

University of CAlifornia, SAn Diego

9500 Gilman Drive \#0112

LA Jolla, CA 92093-0112

E-mail address: astern@math.ucsd.edu

Received 05/26/2009, accepted 01/19/2010

Thanks to Melvin Leok, Jerry Marsden, David Martín de Diego, and Joris Vankerschaver for helpful discussions and feedback during the development of this work, as well as to the editor and anonymous referee for their valuable comments and suggestions. The research was supported in part by NSF (PFC Award 0822283 and DMS Award 0715146), as well as by NIH, HHMI, CTBP, and NBCR. 